\title{
Long term management challenges in diabetic patients with rhino-orbito- -cerebral mucormycosis
}

\begin{abstract}
There has been a recent upsurge in prevalence of diabetes mellitus in developing world. This has resulted in exponential increase in the incidence of both communicable and non-communicable diseases. Some of the infections increase morbidity and mortality associated with diabetes. Rhino-orbital-cerebral mucormycosis (ROCM) is one of the fatal opportunistic infections in diabetes. There is little data published regarding the short and long term management of patients suffering from this invasive fungal infection. Hereby, we report few cases of ROCM with varied presentations and their short and long term follow up. These patients were initially treated with injectable amphotericin B and later followed up with oral posaconazole. (Clin Diabetol 2020; 9; 2: 138-140)
\end{abstract}

Key words: diabetes, posaconazole, rhino-orbital-cerebral mucormycosis

\section{Introduction}

As incidence of diabetes is rising globally, infections and sepsis are also imposing enormous burden on the disease outcome [1]. Mucormycosis is relatively uncommon and frequently fatal angio-invasive fungal

Address for correspondence:

Dr SV Madhu

Centre for Diabetes Endocrinology and Metabolism

University College of Medical Sciences and GTB Hospital

Dilshad Garden, Delhi-110095, India

Phone:+91-11-22596438

e-mail: drsvmadhu@gmail.com

Clinical Diabetology 2020, 9, 2, 138-140

DOI: $10.5603 /$ DK.2020.0005

Received: 30.10 .2019

Accepted: 26.12 .2019 infection. It usually develops in immunocompromised patients and diabetes is a common predisposing factor [2]. Despite aggressive surgical debridement and antifungal therapy, the prognosis of disease is markedly poor. Antifungal agents have poor tissue penetration property so surgical intervention should be considered first for the treatment of invasive fungal infection. Due to need for extensive surgical debridement, role of other treatment modalities should be explored. Here, we describe three patients with ROCM with varied clinical stages, treated with conventional therapy i.e. amphotericin B later posaconazole was started for follow up.

\section{Case report}

A 50 year old male patient who had uncontrolled type 2 diabetes mellitus for one and a half years presented with high fever for 10 days along with pain and swelling in left side of face and eye. Later, patient developed diplopia and unilateral decrease in vision for five days followed by complete loss of vision for last two days. It was accompanied by difficulty in swallowing specially for solids, non bilious vomiting and change in voice. General physical examination was normal. At the time of examination, patient was conscious with oral temperature of $39.8^{\circ} \mathrm{C}$, a blood pressure of $140 / 90 \mathrm{~mm} \mathrm{Hg}$ and a pulse of $116 / \mathrm{min}$. Vision and ocular movements in the right eye was normal but the left eye showed negative perception of light. Pupils were fixed and dilated. Ophthalmoplegia was present. Movements were restricted in all directions. Initial assessment showed leucocytosis with raised ESR and slightly decreaased kidney function test. The initial $\mathrm{HbA}_{1 c}$ was $13.6 \%$ on oral hypoglycemic regimen. Chest $X$ ray was normal. ENT examination showed blackish 
ulcerating growth in middle meatus. Potassium hydroxide mount $(\mathrm{KOH})$ proved mucormycosis and later contrast enhanced MRI revealed left rhino-orbito-cerebral mucormycosis with cavernous sinus thrombosis. Involvement of left sided ethmoid, maxillary and frontal sinus was also present. There was intraorbital extension into medial orbit, medial rectus, orbital apex, inferior orbital fissure with slight extension into infratemporal fossa. Left cavernous sinus appeared bulky. Patient was started on liposomal amphotericin B along with broad spectrum antibiotics. Diabetes was controlled with multi dosage insulin regimen (on a dose of approx 0.9 units per kg body weight). Amphotericin B was started at a dose of $3 \mathrm{mg} / \mathrm{kg}$ which was escalated to $5 \mathrm{mg} / \mathrm{kg}$ and continued for 6 weeks. No surgical intervention was done. Repeated ENT and ophthalmological consultation was sought. Repeat MRI was done after 6 weeks of injectable therapy that showed no further progression although there was reduced bulkiness in the cavernous sinus. Clinically, ophthlmoplegia and restriction of eye ball movement completely recovered with slight improvement in vision over the time. Patient was put on oral posaconazole therapy at a dose of $800 \mathrm{mg}$ per day and 4 months down the line, patient showed clinical improvement with no signs of radiological worsening.

Another patient, 50 years old diabetic female presented with painful swelling in the left retro-orbital region and nose for five days along with fever and progressive breathing difficulty. On admission, the patient was conscious with oral temperature of $39.4^{\circ} \mathrm{C}$, a blood pressure of $120 / 80 \mathrm{~mm} \mathrm{Hg}$ and a pulse of $130 / \mathrm{min}$. She was unable to open the left eye and her vision and left pupillary light reflex were lost. There was leucocytosis, ketonemia with normal liver function, renal function and electrolytes. Blood gasometry confirmed metabolic acidosis. The initial $\mathrm{HbA}_{1 \mathrm{c}}$ was $12.9 \%$. Patient was managed as per diabetic ketoacidosis (DKA) protocol and ENT consultation was sought which was suggestive of extensive black necrotic tissue in the nasal cavity. The patient's nasal structure was completely destroyed and the hard palate was perforated. $\mathrm{KOH}$ mount was suggestive of mucormycosis. Patient was started on liposomal amphotericin B along with broad spectrum antibiotics. Liposomal amphotericin B was started at a dose of $3 \mathrm{mg} / \mathrm{kg}$ which was escalated to $5 \mathrm{mg} / \mathrm{kg}$ and continued for 8 weeks. Diabetes was initially controlled with multi dosage insulin regimen (on a dose of approx 1.2 units per $\mathrm{kg}$ body weight) and later stabilized on approx 0.75 units per kg body weight. Extensive surgical debridement was done. Patient improved clinically and was discharged with no active residual apparent disease. On follow up, her clinical status remained fair.
However, this patient died in the emergency room due to severe pneumonia, respiratory distress with septic shock after one and half years.

The third patient, a 50 years old female with type 2 diabetes for 10 years presented with painful swelling of the right side of face and right eye for one and a half months. On admission, the patient was conscious with oral temperature of $39.4^{\circ} \mathrm{C}$, a blood pressure of $130 / 80 \mathrm{~mm} \mathrm{Hg}$ and a pulse of $100 / \mathrm{min}$. She was unable to open the right eye although vision and right pupillary light reflex were normal. There was leucocytosis with normal liver function, renal function and electrolytes. The initial $\mathrm{HbA}_{1 \mathrm{c}}$ was $13.4 \%$. ENT consultation was sought that showed extensive fungal sinus invasion. She was started on basal bolus insulin regimen at a dose of 0.5 units per kilogram body weight but control was finally achieved on 0.7 units per kilogram body weight. Liposomal amphotericin B was started at a dose of $3 \mathrm{mg} / \mathrm{kg}$ which was escalated to $5 \mathrm{mg} / \mathrm{kg}$ and continued for 6 weeks. Surgery was deferred due to extensive intracerebral involvement. As per ENT opinion patient had been put on amphotericin nasal douche. Later, patient was put on oral posaconazole therapy at a dose of $800 \mathrm{mg}$ per day.

\section{Discussion}

Mucormycosis is a fatal opportunistic infection caused by fungi Mucorales, Rhizopus, Mucor and Rhizomucor species. It usually affects immunecompromised or immune suppressed patients [3]. Till date, surgical debridement is the gold standard for the treatment of ROCM. Surgical debridement has its own limitations. There are several issues in the management of ROCM which still remain unclear. Firstly, most of the recommendations advocate surgical debridement as important lifesaving procedure [4]. This option is often limited due to various reasons (extensive and disfiguring surgery). Secondly, there is no consensus about the duration of the amphotericin B treatment despite unanimity that this is always the first line of treatment. There is not enough data to clarify its duration of use and appropriate time of stopping the treatment. Most recommendations mention amphotericin $B$ should be given till there is radiological and clinical improvement [4]. However, it is often seen in clinical practice that radiological recovery beyond a point is minimal and slow in the absence of surgical debridement. This makes it difficult to decide precisely when to stop amphotericin B.

We gave amphotericin B for 6-10 weeks after diagnosis of the patients which was followed by clinical improvement with some radiological recovery (definitely 
no worsening). Long term follow up treatment with oral posaconazole or other oral antifungal agents following long use of injectable AMB is not indicated. Yet we tried oral posaconazole in two patients for six to eight weeks. Now the patients are clinically improved with no definite radiological worsening. This suggests that posaconazole may be useful as a step down therapy after initial amphotericin B treatment. This case report suggests that opportunistic infections are commonly associated with poorly controlled diabetes. Rhino-orbito-cerebral mucormycosis appear to be one of the primary infection among opportunistic infections.

So, the alternative efficacious therapies are being explored. A few experimental reports have shown promising results with posaconazole or ravuconazole but clinical data shows little or no effect [5]. According to a case report, posaconazole with amphotericin B has shown some promising result [6]. In addition to posaconazole, isavuconazole has been approved by FDA [7].

\section{Conclusion}

This data supports the essential and alternative treatment modalities for the treatment of rhino-orbito-cerebral mucormycosis. Oral antifungal agents e.g., posaconazole appears to have promising role as add on therapy in treatment of ROCM.

\section{REFERENCES}

1. Ogurtsova $K$, da Rocha Fernandes JD, Huang $Y$, et al. IDF Diabetes Atlas: Global estimates for the prevalence of diabetes for 2015 and 2040. Diabetes Res Clin Pract. 2017; 128: 40-50, doi: 10.1016/j.diabres.2017.03.024, indexed in Pubmed: 28437734.

2. Guinea J, Escribano $P$, Vena $A$, et al. Increasing incidence of mucormycosis in a large Spanish hospital from 2007 to 2015: Epidemiology and microbiological characterization of the isolates. PLoS One. 2017; 12(6): e0179136, doi: 10.1371/journal. pone.0179136, indexed in Pubmed: 28591186.

3. Peleg AY, Weerarathna T, McCarthy JS, et al. Common infections in diabetes: pathogenesis, management and relationship to glycaemic control. Diabetes Metab Res Rev. 2007; 23(1): 3-13, doi: 10.1002/dmrr.682, indexed in Pubmed: 16960917.

4. Giudice G, Cutrignelli DA, Sportelli $P$, et al. Rhinocerebral mucormycosis with orosinusal involvement: diagnostic and surgical treatment guidelines. Endocr Metab Immune Disord Drug Targets. 2016; 16(4): 264-269, doi: 10.2174/1871530316666161223145 055, indexed in Pubmed: 28017141.

5. Pfaller MA, Messer SA, Hollis RJ, et al. SENTRY Participants Group, SENTRY Participants Group. Antifungal activities of posaconazole, ravuconazole, and voriconazole compared to those of itraconazole and amphotericin B against 239 clinical isolates of Aspergillus spp. and other filamentous fungi: report from SENTRY Antimicrobial Surveillance Program, 2000. Antimicrob Agents Chemother 2002; 46(4): 1032-1037, doi: 10.1128/aac.46.4.1032-1037.2002, indexed in Pubmed: 11897586.

6. Yoon YK, Kim MJa, Chung YGu, et al. Successful treatment of a case with rhino-orbital-cerebral mucormycosis by the combination of neurosurgical intervention and the sequential use of amphotericin B and posaconazole. J Korean Neurosurg Soc. 2010; 47(1): 74-77, doi: 10.3340/jkns.2010.47.1.74, indexed in Pubmed: 20157385.

7. Whaley SG, Berkow EL, Rybak JM, et al. Azole Antifungal Resistance in and Emerging Non- Species. Front Microbiol. 2016; 7: 2173, doi: 10.3389/fmicb.2016.02173, indexed in Pubmed: 28127295 